\section{Epidermal Growth Factor Receptor Expression Analysis in Different Racial Glioma Patients}

\section{Zhengquan Zhu' ${ }^{1 *}$, Ji Zhang ${ }^{2 \star}$, Zhihuan Zhou ${ }^{2 *}$, Xiaoli Wang ${ }^{3}$, Yanxia $\mathrm{Li}^{4}$, Xin Yang ${ }^{5}$, Cong $\mathrm{Li}^{6 *}$ and Hai Cheng $\mathrm{Xia}^{1 *}$}

${ }^{1}$ Department of neurosurgery, Tumor Hospital Affiliated of Xinjiang Medical University, URUMQI, China

${ }^{2}$ Department of Neurosurgery, State Key Laboratory of Oncology in South China, Collaborative Innovation Center for Cancer Medicine, Sun Yat-sen University Cancer Center, Guangzhou, China

${ }^{3}$ Department of general surgery, Shang Jin Nan Fu Hospital of West China Hospital of Sichuan University, Cheng du, China

${ }^{4}$ Rehabilitation department, the second affiliated hospital of Xinjiang medical university, URUMQI, China

${ }^{5}$ Anesthetic operation center, West China Hospital, Sichuan University, Chengdu, China

${ }^{6}$ Department of colorectal surgery, State Key Laboratory of Oncology in South China, Collaborative Innovation Center for Cancer Medicine, Sun Yat-sen University Cancer Center, Guangzhou, 510060, China

${ }^{*}$ Contributed equally

\section{Abstract}

\section{Background}

Vascular Endothelial Growth Factor (VEGF) represents a promising anti-neoplastic target. VEGF expression in same pathological grade of glioma in different ethnicities patients has not been integrated into clinical practice yet. The aim of our study is to investigate the relationship between VEGF expression level and prognosis in different ethnicities patients with glioma of an identical pathological grade.

*Corresponding authors: Cong Li, Department of colorectal surgery, State Key Laboratory of Oncology in South China, Collaborative Innovation Center for Cancer Medicine, Sun Yat-sen University Cancer Center, Guangzhou, China, E-mail: licong1@sysucc.org.cn

Hai Cheng Xia, Department of Neurosurgery, The Affiliated Tumor Hospital of Xinjiang Medical University, Urumqi, Xinjiang, China, Tel: +86 018999869297 E-mail: xiahaicheng@qq.com

Citation: Zhu Z, Zhang J, Zhou Z, Wang X, Li Y, et al. (2019) Epidermal Growth Factor Receptor Expression Analysis in Different Racial Glioma Patients. J Addict Addictv Disord 6: 29.

Received: July 10, 2019; Accepted: August 01, 2019; Published: August 09, 2019

Copyright: () 2019 Zhu Z, et al. This is an open-access article distributed under the terms of the Creative Commons Attribution License, which permits unrestricted use, distribution, and reproduction in any medium, provided the original author and source are credited.

\section{Methods}

We retrospectively analyzed VEGF expression level by immunohistochemical staining and prognosis in both Chinese Uygur and Han patients with glioma.

\section{Results}

The rate of positive expression of VEGF was $81.97 \%$ in $6 \mathrm{I} \mathrm{Han}$ patients and was $60.61 \%$ in 33 Uygur patients with glioma. There was a significant difference between ethnicity and the VEGF expression $(P=0.023)$. Regarding the impact of VEGF expression level on the prognosis of patients from the two ethnicities, the difference exerted an influence on the survival of patients with Low Grade Glioma ( $L G G)(P>0.05)$, which, however, was associated with the survival of patients with High Grade Glioma (HGG) $(P<0.05)$.

\section{Conclusion}

VEGF expression differs in Han and Uygur patients with glioma. Ethnicity is one factor that has an effect on survival between Uygur and $\mathrm{Han}$ patients with $\mathrm{HGG}$.

Keywords: Ethnicity; Glioma; Prognosis; Vascular endothelial growth factor

\section{Introduction}

Glioma is the most common primary tumors of the brain in adults [1]. The 5-year survival rate in patients with glioma is surprisingly low [2]. Conventional therapies play an important role in the treatment of malignant gliomas. However, the prognosis for patients with malignant gliomas still leaves much to be desired. In recent years, there have been some renewed efforts to develop the novel treatments based on molecular targets of glioma. The identification of these factors that can predict survival is an important goal for treatment of these patients, and a large number of studies have shown that the expression of VEGF was obviously related to the pathological grade of the tumor [3-7]. However, in a review of the literatures, no studies reported the association between VEGF expression level and different ethnic populations with an identical pathological classification. In this study, we compared the difference in VEGF expression level in glioma patients with identical classifications who settled in the Xinjiang province of China to elucidate whether ethnicity affects the VEGF expression and prognosis of these patients.

\section{Material and Methods}

\section{Patients}

This study was approved by the institutional ethical committee and involved 94 paraffin embedded tumor tissue specimens from the affiliated Tumor Hospital of Xinjiang Medical University from January 2005 to December 2010. There were 65 males and 29 females, including 61 Han cases and 33 Uygur cases. The inclusion criteria for the study were newly diagnosed cases without anti-tumor treatment. Patient characteristics were recorded consisting of ethnicity, age, gender, KPS scale, pathological grade and VEGF expression level. All samples were selected from individuals receiving routine treatment 
Citation: Zhu Z, Zhang J, Zhou Z, Wang X, Li Y, et al. (2019) Epidermal Growth Factor Receptor Expression Analysis in Different Racial Glioma Patients. J Addict Addictv Disord 6: 29.

in our hospital with no history of other cancer and no symptoms of other forms of acute or chronic inflammation. The median age was 44 years (range 18 75). Based on the World Health Organization (WHO) classification of tumors of the central nervous system in 2000, there were 6 cases of grade I, 28 cases of grade II, 35 cases of grade III, and 25 cases of grade IV.

\section{Methods}

Tumor specimens were tested by immunohistochemistry for VEGF expression level using the Dako Epidermal Growth Factor Receptor (EGFR) pharm Dx assay ${ }^{\mathrm{TM}}$ (Dako, Denmark). VEGF expression level immunostaining score was calculated as the percentage of positively stained tumor cells and the staining intensity. A nucleus dyeing rate of $0 \sim 9 \%$ was marked as,$+ 10 \% \sim 49 \%[2+]$ and $>50 \%[3+]$ [8]. Both the percentage of positive cells and the staining intensity were evaluated under double-blind conditions. Two independent pathologists examined and scored each sample without any knowledge of the pathological outcome. The VEGF expression score was calculated as the percentage positive score $\times$ the staining intensity score and ranged from 0 to 9 .

\section{Statistical analysis}

SPSS 20.0 was used in all statistical analyses. Numerical variables were summarized as an mean (standard deviation) or median (interquartile range). For comparison between the two groups, categorical variables were analyzed via the chi-square test. To elaborate the relationship between glioma VEGF expression level and pathological grades in the two ethnicities, the contingency table test was employed. Significance was defined as a $\mathrm{P}$ value of $<0.05$.

\section{Results}

\section{VEGF expression level in Uygur and Han glioma patients}

Positive VEGF expression rate in the tumor tissues in Han and Uygur glioma patients were $81.97 \%$ and $60.61 \%$, respectively. When the positive rate of VEGF expression in the two groups was compared and analyzed, the expression difference was statistically significant $(\mathrm{P}=0.023)$. The VEGF expression level in Uygur patients was obviously lower than that in Han patients, which demonstrated that ethnicity can affect VEGF expression in glioma patients (Table 1).

\begin{tabular}{|c|c|c|c|}
\hline \multirow{2}{*}{ Race } & \multicolumn{2}{|c|}{ VEGF } & \multirow{2}{*}{ Number } \\
\cline { 2 - 3 } & Positive & Negative & \\
\hline Han & $50(81.97 \%)$ & $11(18.03 \%)$ & 61 \\
\hline Uygur & $20(60.61 \%)$ & $13(39.39 \%)$ & 33 \\
\hline Total & 70 & 24 & 94 \\
\hline
\end{tabular}

Table 1: The positive rate of theVEGF expression in Uighur and Han glioma patients.

\section{The correlation between the pathological grade and VEGF expression level in Han glioma patients}

We analyzed the association between pathological grade and VEGF expression level in Han glioma patients. The result showed that the VEGF expression level in Han glioma patients was significantly related with tumor pathological classification $(\mathrm{P}=0.024)$ (Table 2).

\begin{tabular}{|c|c|c|c|c|c|}
\hline \multirow{2}{*}{$\begin{array}{c}\text { Pathological } \\
\text { Grade }\end{array}$} & \multicolumn{2}{|c|}{ VEGF Expression of Han Glioma Patients } & \multirow{2}{*}{ Number } \\
\cline { 2 - 5 } & Negative & + & ++ & +++ & \\
\hline Grade I & $1(50 \%)$ & $1(50 \%)$ & $0(0 \%)$ & $0(0 \%)$ & 2 \\
\hline Grade II & $2(10 \%)$ & $12(60 \%)$ & $5(25 \%)$ & $1(5 \%)$ & 20 \\
\hline Grade III & $6(26.1 \%)$ & $5(10 \%)$ & $9(21.7 \%)$ & $3(9.2 \%)$ & 23 \\
\hline Grade IV & $2(12.5 \%)$ & $3(18.8 \%)$ & $4(25 \%)$ & $7(43.6 \%)$ & 16 \\
\hline Total & 11 & 21 & 18 & 11 & 61 \\
\hline
\end{tabular}

Table 2: The correlation between pathologic grade and VEGF expression level in Han patients.

\section{The correlation between the pathological grade and VEGF expression level in Uygur glioma patients}

Table 3 displays the relationship between the pathological grade and VEGF expression level of 33 Uygur glioma patients. The results indicated that the VEGF expression level in Uygur glioma patients was not significantly different from the tumor pathological grade $(\mathrm{P}=0.683)$.

\begin{tabular}{|c|c|c|c|c|c|}
\hline \multirow{2}{*}{$\begin{array}{c}\text { Pathological } \\
\text { Grade }\end{array}$} & \multicolumn{4}{|c|}{ VEGF Expression of Uygur Glioma Patients } & \multirow{2}{*}{ Number } \\
\cline { 2 - 5 } & Negative & + & ++ & +++ & \\
\hline Grade I & $1(25 \%)$ & $1(25 \%)$ & $2(50 \%)$ & $0(0 \%)$ & 4 \\
\hline Grade II & $4(50 \%)$ & $1(12.5 \%)$ & $2(25 \%)$ & $1(12.5 \%)$ & 8 \\
\hline Grade III & $4(33.3 \%)$ & $5(41.4 \%)$ & $2(16.7 \%)$ & $1(8.3 \%)$ & 12 \\
\hline Grade IV & $4(44.4 \%)$ & $1(11.1 \%)$ & $4(44.4 \%)$ & $0(0 \%)$ & 9 \\
\hline Total & 13 & 8 & 10 & 2 & 33 \\
\hline
\end{tabular}

Table 3: The correlation between pathologic grade and VEGF expression level in Uygur patients.

\section{Survival analysis between Uygur and Han glioma patients with identical pathological grade}

In patients with grade I and II glioma, the median survival was62 months in Han people (22 cases) and 69 months in Uygur people (9 cases). There was no statistical difference between the two groups $(\mathrm{P}=0.125)$, namely, the survival time of LGG patients has no obvious relationship with ethnicity (Figure 1).

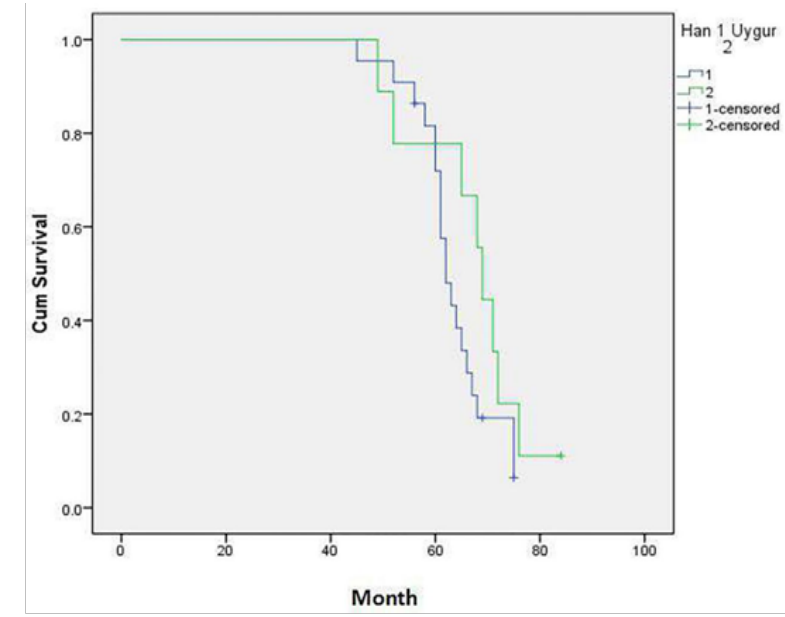

Figure 1: The survival timeshows no statistically significant difference between Uygur and Han glioma patients with low-grade glioma. 
In glioma patients with grade III, the median survival was 16.2 months in the Han group ( 25 cases) and 28.7 months in the Uygur group (10 cases). There was a significant difference between the two groups $(\mathrm{P}=0.007)$. The average survival time of Uygur patients with grade III glioma was longer than that of Han patients (Figure 2).

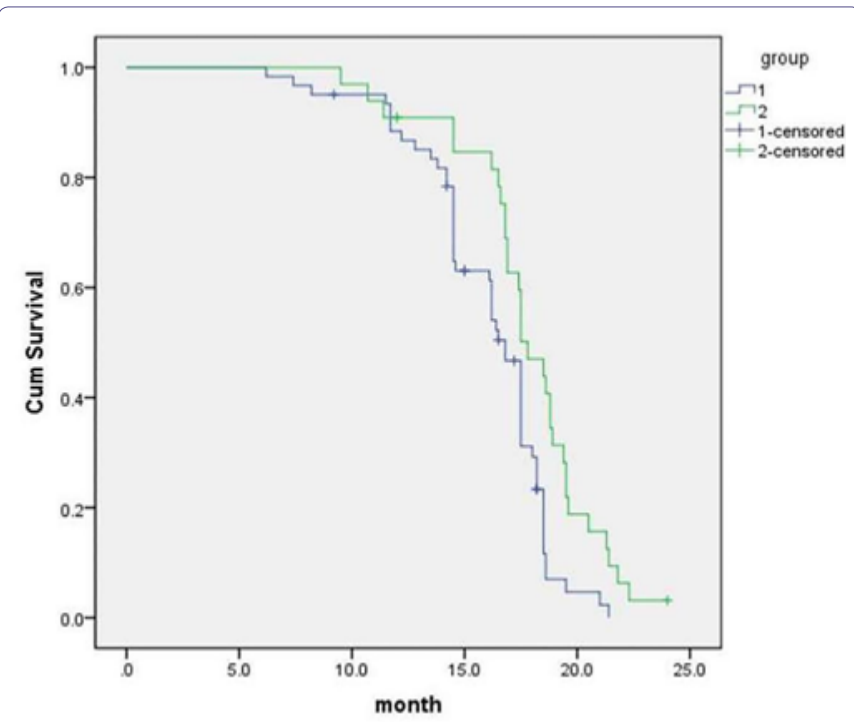

Figure 2: The survival timein Uygur patients is longer than that in Han patients with grade III glioma.

In patients with grade IV glioma, the median survival time was 11.8 months in the Han group (14 cases) and 17.5 months in the Uygur group (13 cases).There was a significant difference between the two groups $(\mathrm{P}=0.007)$. The survival time of Uygur glioma patients with grade IV glioma was longer that of Han patients (Figure 3).

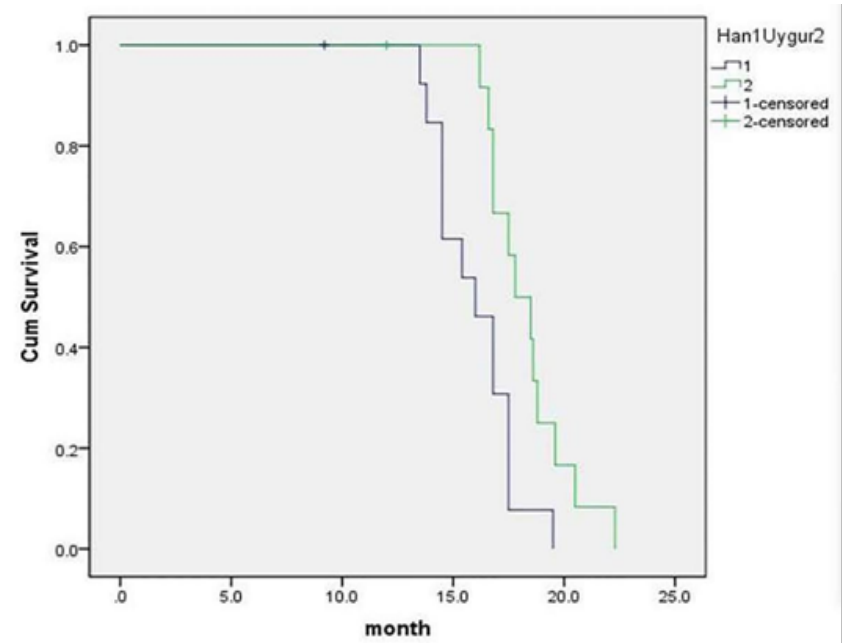

Figure 3: The survival timein Uygur patients is longer than that in Han patients with grade IV glioma.

\section{Discussion}

Vascular endothelial growth factor can induce angiogenesis, increase vascular permeability and promote division and proliferation of vascular endothelial cell [9]. VEGF also plays a key role in the process of tumor growth [10]. The vascular endothelial growth factor family of polypeptide growth factors regulates a family of VEGF receptor tyrosine kinases with pleiotropic downstream effects [4]. Angiogenesis is the best known of these effect Angiogenesis leads to new blood vessel formation and is implicated in both physiological and pathological situations [7]. The vascular endothelial growth factor family is the major mediator of this process. in patients with classical Hodgkin lymphoma (cHL), the level of VEGF-A, VEGFR-1 and VEGFR-2 was tested, only the expression of VEGFR-2 was positively correlated with serum albumin levels $\geq 4 \mathrm{~g} / \mathrm{dL}$. No correlation with patient outcome was observed in CHL patients [4]. The T allele of VEGF $+936 \mathrm{C} / \mathrm{T}$ polymorphism is more common in primary tumors of the glioma, but there is no statistical relation with survival [5]. Angiogenesis commonly attributed to the anticrime and paracrine production of VEGF-A, which up regulates the VEGF signal transduction pathway, is a prominent feature of glioblastoma [11]. VEGF is secreted more in the glioma tissue than in normal brain tissue $[12,13]$. In recent years, the targeted molecular therapy of glioma based on VEGF as the target has gradually become a new strategy [14]. A large number of studies have indicated that VEGF expression level in glioma was closely related to the degree of malignancy of the tumor [15]. The higher the tumor pathological grade is, the higher the level of VEGF expression is [16,17]. Sometimes, The level of expression of some tumor related factors in tumors is closely related to the malignancy of tumors and the prognosis of tumors in Chinese just as Neutrophil-to-Lymphocyte Ratio (NLR) and Platelet-To-Lymphocyte Ratio (PLR) in the glioma, increased preoperative NLR and PLR are associated with worse OS, and NLR may be an independent risk factor to identify glioma patients with poor prognosis [18].

In the present study, we performed VEGF immunohistochemical staining on the embedded paraffin specimens. The positive rate of VEGF expression in Han patients was significantly correlated with tumor pathological grade, which was by and large consistent with other studies [19]. However, the difference was that positive VEGF expression rate in Uygur patients was remarkably lower than that in Han patients. Nonetheless, there was no obvious relationship between pathological grade in the Uygur glioma patients and the VEGF expression level, but the pathological grade was positively associated with VEGF expression level. We surmised that the negative result was possibly associated with the limited number of Uygur patients. Additionally, the VEGF expression level in HGG was distinctly high compared with that in LGG, which implied that the VEGF expression level may be considered as an index of the glioma grade.

When the ethnicity factor was analyzed for patients with gliomas of different pathological grades, there was no obvious difference regarding the role of ethnicity in the survival of LGG patients. However, this difference was evident between Han and Uygur patients with HGG. Thus, ethnicity is one factor impacting the survival in HGG patients.

Many studies confirmed that VEGF is highly expressed in tumor tissue. Xi observed that the rate of positive VEGF expression was positively correlated with pathological grade, the rate of the positive expression in the higher grade group was higher than that in the lower grade group and the rate of positive expression in the invasive cancer group was higher than that in the superficial tumor group in bladder cancer [20]. These findings indicated that the expression of VEGF might be associated closely with the invasiveness, metastasis and other 
Citation: Zhu Z, Zhang J, Zhou Z, Wang X, Li Y, et al. (2019) Epidermal Growth Factor Receptor Expression Analysis in Different Racial Glioma Patients. J Addict Addictv Disord 6: 29.

biological traits of bladder cancer. Some researchers discovered that the expression of VEGF in esophageal carcinoma was associated with lymphatic metastasis and the depth of invasion, and the expression was lower in para-carcinoma tissues than in esophageal cancer tissues [21]. Many scholars have put forward different opinions about whether the expression of VEGF was related to the clinical characteristics of endometrial diseases. For example, Abulafiao thought there was no correlation between vasculogenesis and the tumor grade, but Nakayama had the opposite opinion [22,23]. They considered that there was no correlation between angiogenesis and other clinical pathological parameters, and that angiogenesis was related to tumor grade, but some study found that Genetic variants were not associated with gliomas. Specific lifestyle habits and comorbidities stood out as independent risk factors for the disease. Low-grade gliomas showed an increase in patient survival with TMZ+RT treatment $[24,25]$.

Similar ethnicity-related target expression was tested for other molecular targets such as the EGFR [7,26]. However, several phase III trials comparing EGFR inhibitor-gefitinib to placebo in advanced non small cell lung cancer patients demonstrated no improvement in overall survival in an unselected population [27,28]. Asian patients achieved a statistically significant improvement in overall survival with gefitinib. Regarding the mechanism, patients who benefited from gefitinib tended to harbor somatic activating mutations in the EGFR gene $[29,30]$. These data illustrated that molecular mutations could be used to identify subgroup of patients to differentiate VEGF expression in Han and Uygur patients. Additionally, initial studies that analyzed the efficacy of bevacizumab with recurrent glioblastoma explained its clinical activity, and first-line use of bevacizumab did not improve overall survival, although progression-free survival was prolonged [30]. As a result, we conferred that the VEGF expression level determined the response of bevacizumab and led to the different efficacy.

There was a significant difference in the correlation of VEGF expression level and tumor pathological grade between Xinjiang Uygur and Han patients with glioma. We reckon that the cause of the difference is due to ethnicity. Due to this inconsistency, further study in a large series is required to determine whether there is a difference in the curative effect of the molecular targeted therapy for glioma based on VEGF as the target in Xinjiang Uygur and Han glioma patients.

\section{Conclusion}

The present study reveals a significant difference in VEGF expression levels in glioma tissue between Uygur and Han glioma patients, although the number of patients in our series is limited and the monitoring method might be responsible for the difference in VEGF expression in glioma tissue. It is crucial to analyze the difference in efficacy of bevacizumab as a targeted VEGF inhibitor in Han and Uygur glioma patients in future.

\section{Abbreviations}

VEGF: Vascular Endothelial Growth Factor;

HGG: High Grade Glioma;

LGG: Low Grade Glioma;

EGFR: Epidermal Growth Factor Receptor.

\section{Acknowledgment}

This work was supported by Project of Guangdong Medical Science and Technology Research Foundation (No. A2018017) and Natural Science Foundation of Xinjiang Uygur Autonomous Region ( No. 2018D01C251).

\section{Conflict of Interest Statement}

Authors state no conflict of interest.

\section{Ethical Approval}

This article does not contain any studies with human participants or animals performed by any of the authors.

\section{References}

1. Mentlein R, Forstreuter F, Mehdorn HM, Held-Feindt J (2004) Functional significance of vascular endothelial growth factor receptor expression on human glioma cells. J Neurooncol 67: 9-18.

2. Guo P, Xu L, Pan S, Brekken RA, Yang ST, et al. (2001) Vascular endothelial growth factor isoforms display distinct activities in promoting tumor angiogenesis at different anatomic sites. Cancer Res 61: 8569-8577.

3. Bao G, Wang M, Guo S, Han Y, Xu G (2011) Vascular endothelial growth factor $+936 \mathrm{C} / \mathrm{T}$ gene polymorphism and glioma risk in a Chinese Han population. Genetic Test Mol Biomarkers 15: 103-106.

4. Dimtsas GS, Georgiadi EC, Karakitsos P, Vassilakopoulos TP, Thymara I, et al. (2014) Prognostic significance of immunohistochemical expression of the angiogenic molecules vascular endothelial growth factor-A, vascular endothelial growth factor receptor-1 and vascular endothelial growth factor receptor-2 in patients with classical Hodgkin lymphoma. Leuk Lymphoma 55: 558-564.

5. Veganzones S, de la Orden V, Requejo L, Mediero B, González ML, et al. (2017) Genetic alterations of IDH1 and Vegf in brain Tumors. Brain Behav 7: 00718.

6. Li R, Zhao Y, Fan W, Chen H, Chen Y, et al. (2011) Possible association between polymorphisms of human vascular endothelial growth factor $\mathrm{A}$ gene and susceptibility to glioma in a Chinese population. Int J Cancer 128: $166-175$.

7. Calastri MCJ, Rodrigues NLTO, Hatori G, Gregório ML, Brancati CIFO, et al. (2018) Genetic variants related to angiogenesis and apoptosis in patients with glioma. Arq Neuropsiquiatr 76: 393-398.

8. Xu LZ, Yang WT (1996) Criteria for determining the results of immunohistochemical reactions. China Oncology 6: 229-231.

9. Kondo S, Matsumoto T, Yokoyama Y, Ohmori I, Suzuki H (1995) The shortest is form of human vascular endothelial growth factor/vascular permeability factor (VEGF/VPF121) produced by Saccharomyces cerevisiae promotes both angiogenesis and vascular permeability. Biophysica Acta 1243: $195-202$.

10. Epstein RJ (2007) VEGF signaling inhibitors: More pro-apoptotic anti-angiogenic. Cancer Metastasis Rev 26: 443-452.

11. Kroll J, Waltenberger J (2000) Regulation of endothelial function and angiogenesis by vascular endothelial growth factor-A (VEGF-A). Z Kardiol 89: 206-218.

12. Wei GQ, GuanY, Zhang GX (2002) The relevant research about peritumoral edema of metastatic encephaloma and the expression of VEGF. Journal of Practical Radiology 18: 282-284.

13. Duan ZX, Xie QL (2010) The role of VEGF in tumor growth and angiogenesis. World Chinese Journal of Digestology 18: 2894-2900. 
Citation: Zhu Z, Zhang J, Zhou Z, Wang X, Li Y, et al. (2019) Epidermal Growth Factor Receptor Expression Analysis in Different Racial Glioma Patients. J Addict Addictv Disord 6: 29.

14. Hofer S, Elandt K, Greil R, Hottinger AF, Huber U, et al. (2011) Clinical outcome with bevacizumab in patients with recurrent high-grade glioma treated outside clinical trials. Acta Oncol 50: 630-635.

15. Grau SJ, Trillsch F, HermsJ, Thon N, Nelson PJ, et al. (2007) Expression of VEGFR3 in glioma endothelium correlates with tumor grade. J Neurooncol 82: 141-150.

16. Bu XY, Zhang X, Yi SY (2001) The relationship between expression of VEGF gene with Glioma angiogenesis and cerebral edema. Chinese Neurosurgical Journal 17: 21-24.

17. Li LJ, Tong JZ, Cui J, Wu JH, Wang HB (2016) Expression and its relationship with the degree of malignancy and prognosis of VEGF, EGFR and PDGF in human brain gliomas. Hainan Med J 27: 2251-2254.

18. Wang J, Xiao W, Chen W (2018) prognostic significance of preoperative neutrophil-to-lymphocyte ratio and platelet-to-lymphocyte ratio in patients with glioma. EXCLI J 28: 505-512.

19. Yang J, Zhao Z, Zhong X (2017) Correlation analysis of the clinic pathological features of glioma and expression of p53 and VEGF. Int J Clin Exp Med 10: 3606-3611

20. Cui X, Li WT (2012) The expression of VEGF and Clusterin in bladder cancer tissue and its correlation analysis. Chinese Journal of cancer surgery 4: 217-219.

21. Poltorak Z, Cohen T, Sivan R (2007) VEGF145, a secreted vascular en dothelial growth factor that binds to extraeellular matrix. Biol Chem 272 . 7151-7158.

22. Abulafia O, Ruiz JE, Holcomb K, Dimaio TM, Lee YC, et al. (2000) Angiogenesis in early invasive and low-malignant-potential epithelial ovarian carcinoma. Obstet Gynecol 95: 548-552.
23. Nakayama K, Kanzaki A, Takebayashi Y, Toi M, Bando H, et al. (2001) Different features of angiogenesis between ovarian and breast carcinoma. Cancer Lett 170: 161-167.

24. Mitsudomi T, Yatabe Y (2007) Mutations of the epidermal growth factor receptor gene and related genes as determinants of epidermal growth factor receptor tyrosine kinase inhibitors sensitivity in lung cancer. Cancer Sci 98: 1817-1824.

25. ZhangY, Sun Y, Pan Y, Li C, Shen L, et al. (2012) Frequency of driver mutations in lung adenocarcinoma from female never-smokers varies with histologic subtypes and age at diagnosis. Clin Cancer Res 18: 1947-1953.

26. Fukuoka M, Yano S, Giaccone G, Tamura T, Nakagawa K, et al. (2003) Multi-institutional randomized phase II trial of gefitinib for previously treated patients with advanced non-small-cell lung cancer. J Clin Oncol 21: $2237-2246$

27. Ostrom QT, Gittleman H, Liao P, Rouse C, Chen Y, et al. (2014) CBTRUS statistical report: primary brain and central nervous system tumors diagnosed in the United States in 2007-2011. Neuro Oncol 16: 1-63.

28. Lynch TJ, Bell DW, Sordella R, Gurubhagavatula S, Okimoto RA, et al. (2004) Activating mutations in the epidermal growth factor receptor underlying responsiveness of non-small-cell lung cancer to gefitinib. N Engl J Med 350: 2129-2139.

29. Stamos J, Sliwkowski MX, Eigenbrot C (2002) Sreucture of the epidermal growth factor receptor kinase domain alone and in complex with a 4-anilinoquinazoline inhibitor. J Biol Chem 77: 46265-46272.

30. Gilbert MR, Dignam JJ, Armstrong TS, Wefel JS, Blumenthal DT, et al. (2014) A randomized trial of bevacizumab for newly diagnosed glioblastoma. N Engl J med 370: 699-708. 


\section{II}

Journal of Anesthesia \& Clinical Care

Journal of Addiction \& Addictive Disorders

Advances in Microbiology Research

Advances in Industrial Biotechnology

Journal of Agronomy \& Agricultural Science

Journal of AIDS Clinical Research \& STDs

Journal of Alcoholism, Drug Abuse \& Substance Dependence

Journal of Allergy Disorders \& Therapy

Journal of Alternative, Complementary \& Integrative Medicine

Journal of Alzheimer's \& Neurodegenerative Diseases

Journal of Angiology \& Vascular Surgery

Journal of Animal Research \& Veterinary Science

Archives of Zoological Studies

Archives of Urology

Journal of Atmospheric \& Earth-Sciences

Journal of Aquaculture \& Fisheries

Journal of Biotech Research \& Biochemistry

Journal of Brain \& Neuroscience Research

Journal of Cancer Biology \& Treatment

Journal of Cardiology: Study \& Research

Journal of Cell Biology \& Cell Metabolism

Journal of Clinical Dermatology \& Therapy

Journal of Clinical Immunology \& Immunotherapy

Journal of Clinical Studies \& Medical Case Reports

Journal of Community Medicine \& Public Health Care

Current Trends: Medical \& Biological Engineering

Journal of Cytology \& Tissue Biology

Journal of Dentistry: Oral Health \& Cosmesis

Journal of Diabetes \& Metabolic Disorders

Journal of Dairy Research \& Technology

Journal of Emergency Medicine Trauma \& Surgical Care

Journal of Environmental Science: Current Research

Journal of Food Science \& Nutrition

Journal of Forensic, Legal \& Investigative Sciences

Journal of Gastroenterology \& Hepatology Research

Journal of Gerontology \& Geriatric Medicine
Journal of Genetics \& Genomic Sciences

Journal of Hematology, Blood Transfusion \& Disorders

Journal of Human Endocrinology

Journal of Hospice \& Palliative Medical Care

Journal of Internal Medicine \& Primary Healthcare

Journal of Infectious \& Non Infectious Diseases

Journal of Light \& Laser: Current Trends

Journal of Modern Chemical Sciences

Journal of Medicine: Study \& Research

Journal of Nanotechnology: Nanomedicine \& Nanobiotechnology

Journal of Neonatology \& Clinical Pediatrics

Journal of Nephrology \& Renal Therapy

Journal of Non Invasive Vascular Investigation

Journal of Nuclear Medicine, Radiology \& Radiation Therapy

Journal of Obesity \& Weight Loss

Journal of Orthopedic Research \& Physiotherapy

Journal of Otolaryngology, Head \& Neck Surgery

Journal of Protein Research \& Bioinformatics

Journal of Pathology Clinical \& Medical Research

Journal of Pharmacology, Pharmaceutics \& Pharmacovigilance

Journal of Physical Medicine, Rehabilitation \& Disabilities

Journal of Plant Science: Current Research

Journal of Psychiatry, Depression \& Anxiety

Journal of Pulmonary Medicine \& Respiratory Research

Journal of Practical \& Professional Nursing

Journal of Reproductive Medicine, Gynaecology \& Obstetrics

Journal of Stem Cells Research, Development \& Therapy

Journal of Surgery: Current Trends \& Innovations

Journal of Toxicology: Current Research

Journal of Translational Science and Research

Trends in Anatomy \& Physiology

Journal of Vaccines Research \& Vaccination

Journal of Virology \& Antivirals

Archives of Surgery and Surgical Education

Sports Medicine and Injury Care Journal

International Journal of Case Reports and Therapeutic Studies

Submit Your Manuscript: http://www.heraldopenaccess.us/Online-Submission.php 